\title{
LIQUID DROP MODEL FOR NUCLEAR MATTER IN THE DILUTE LIMIT*
}

\author{
LUKAS EMMERT ${ }^{\dagger}$, RUPERT L. FRANK ${ }^{\ddagger}$, AND TOBIAS KÖNIG ${ }^{\dagger}$
}

\begin{abstract}
We consider the liquid drop model for nuclei interacting with a neutralizing homogeneous background of electrons. The regime we are interested in is when the fraction between the electronic and the nuclear charge densities is small. We show that in this dilute limit the thermodynamic ground state energy is given to leading order by that of an isolated nucleus.
\end{abstract}

Key words. liquid drop model, nuclear pasta, Coulomb system

AMS subject classifications. 49J40, 49S05, 81V35

DOI. $10.1137 / 19 \mathrm{M} 1274420$

1. Introduction and main theorem. Gamow's liquid drop model [9] is a simple model in nuclear physics which has recently attracted a lot of attention in mathematics; see, for instance, $[4,5,2,13,15,19,7,16,3,14,8]$.

We begin with the description of a single nucleus in this model. Possible shapes of a nucleus are (measurable) sets $\Omega \subset \mathbb{R}^{3}$, and their measure $|\Omega|$ is interpreted as the number of nucleons in suitable units. The energy of such a nucleus is, again in suitable units,

$$
\mathcal{E}[\Omega]:=\operatorname{Per}(\Omega)+\frac{1}{2} \int_{\Omega} \int_{\Omega} \frac{\mathrm{d} x \mathrm{~d} y}{|x-y|},
$$

where $\operatorname{Per}(\Omega)$ denotes the perimeter in the sense of De Giorgi of a set $\Omega$. This leads to the variational problem of finding, for a given $A>0$,

$$
E(A):=\inf \left\{\mathcal{E}[\Omega]: \Omega \subset \mathbb{R}^{3},|\Omega|=A\right\} .
$$

It is known [7] (see also [16]) that there is an $A^{*}>0$ such that $\frac{E\left(A^{*}\right)}{A^{*}}=\inf _{A>0} \frac{E(A)}{A}$ and that there is a minimizing set $\Omega^{*} \subset \mathbb{R}^{3}$ with $\left|\Omega^{*}\right|=A^{*}$ such that $\mathcal{E}\left[\Omega^{*}\right]=E\left(A^{*}\right)$. This set $\Omega^{*}$ is strongly conjectured, but not known, to be a ball. Physically, it corresponds to a nucleus with the greatest binding energy per nucleon, which is a certain isotope of nickel.

In this paper, we are interested in a system consisting of a large number of nuclei interacting with a uniform background of electrons, as arises, for instance, in the crust of a neutron star. As usual in statistical mechanics, we confine the system to a box $[-L / 2, L / 2]^{3}$ and are interested in the thermodynamic limit $L \rightarrow \infty$. For finite $L>0$, allowed nuclear configurations are described by measurable sets $\Omega \subset[-L / 2, L / 2]^{3}$,

\footnotetext{
* Received by the editors July 12, 2019; accepted for publication (in revised form) February 12, 2020; published electronically April 22, 2020.

https://doi.org/10.1137/19M1274420

Funding: The work of the second author was partially supported by the National Science Foundation grant DMS-1363432.

$\dagger$ Mathematisches Institut, LMU Munich, Munich, 80333, Germany (lukas.emmert@lmu.de, tkoenig@math.lmu.de).

${ }_{\ddagger}^{\ddagger}$ Mathematisches Institut, LMU Munich, Munich, 80333, Germany, and Department of Mathematics, California Institute of Technology, Pasadena, CA 91125 (rlfrank@caltech.edu).
}

1980 
and their energy is

$$
\mathcal{E}_{\vartheta, L}[\Omega]:=\operatorname{Per}(\Omega)+\frac{1}{2} \int_{[-L / 2, L / 2]^{3}} \int_{[-L / 2, L / 2]^{3}}\left(1_{\Omega}(x)-\vartheta\right) \frac{1}{|x-y|}\left(1_{\Omega}(y)-\vartheta\right) \mathrm{d} x \mathrm{~d} y .
$$

The parameter $\vartheta \in(0,1]$ here describes the quotient between the electron and the nucleon charge density. We are interested in the ground state energy

$$
E_{\vartheta, L}:=\inf \left\{\mathcal{E}_{\vartheta, L}[\Omega]: \Omega \subset[-L / 2, L / 2]^{3},|\Omega|=\vartheta L^{3}\right\} .
$$

Note that the constraint $|\Omega|=\vartheta L^{3}$ means that we only consider neutral configurations.

Our main result concerns the behavior of the energy per unit volume, $E_{\vartheta, L} / L^{3}$, in the dilute limit $\vartheta \rightarrow 0$. The crucial point is to establish this uniformly in $L$.

THEOREM 1.1 (Ground state energy asymptotics). There is a constant $C>0$ such that the following bounds hold.

(i) For all $\vartheta \in\left(0, \frac{1}{2}\right]$ and $L>0$ such that $\vartheta^{1 / 3} L \geq C$, we have

$$
\frac{E_{\vartheta, L}}{\vartheta L^{3}} \leq \frac{E\left(A^{*}\right)}{A^{*}}+C \vartheta^{1 / 3}+\frac{C}{\vartheta^{1 / 3} L} .
$$

(ii) For all $\vartheta \in(0,1]$ and $L>0$, we have

$$
\frac{E_{\vartheta, L}}{\vartheta L^{3}} \geq \frac{E\left(A^{*}\right)}{A^{*}}-C \vartheta^{1 / 5} .
$$

Remark 1.2. Our result implies, in particular, that the thermodynamic limit

$$
e(\vartheta):=\lim _{L \rightarrow \infty} L^{-3} E_{\vartheta, L}
$$

satisfies

$$
e(\vartheta)=\vartheta\left(\frac{E\left(A^{*}\right)}{A^{*}}+o(1)\right) \quad \text { as } \vartheta \rightarrow 0 .
$$

Remark 1.3. The bounds in Theorem 1.1 give the asymptotics of the energy for $\vartheta$ close to 0 . By a simple symmetry argument our theorem yields analogous asymptotics for $\vartheta$ close to 1 . Namely, for $\vartheta \in\left[\frac{1}{2}, 1\right)$ and $(1-\vartheta)^{1 / 3} L \geq C$, we have

$$
-C(1-\vartheta)^{1 / 5}-\frac{6}{L} \leq \frac{E_{\vartheta, L}}{(1-\vartheta) L^{3}}-\frac{E\left(A^{*}\right)}{A^{*}} \leq C(1-\vartheta)^{1 / 3}+\frac{C}{(1-\vartheta)^{1 / 3} L} .
$$

This follows from the fact that we have for $\Omega \subset Q_{L}$

$$
\mathcal{E}_{\vartheta, L}[\Omega]=\mathcal{E}_{1-\vartheta, L}\left[Q_{L} \backslash \Omega\right]-\left(\mathcal{H}^{2}\left(\partial Q_{L}\right)-2 \mathcal{H}^{2}\left(\partial Q_{L} \cap \bar{\Omega}\right)\right),
$$

where the closure of $\Omega$ is taken in the measure theoretic sense. The term in parentheses is bounded in absolute value by $6 L^{2}$. Therefore, (1.8) follows from the bounds in Theorem 1.1.

Remark 1.4. The power $1 / 5$ of $\vartheta$ in (1.6) is technical. It is an interesting question to decide whether the power $1 / 3$ in (1.5) is best possible. The assumption $\vartheta^{1 / 3} L \geq$ $C$ and the corresponding remainder term in (1.5) are not severe restrictions in the thermodynamic limit and are imposed mainly for a simple statement. 
Theorem 1.1 significantly improves a result of Knüpfer, Muratov, and Novaga [16], who show a similar asymptotic equality in the ultra-dilute limit $\vartheta \sim L^{-2}$, where the background density vanishes in the limit $L \rightarrow \infty$. In contrast, we can perform first the limit $L \rightarrow \infty$ and then $\vartheta \rightarrow 0$. In the regime $\vartheta \sim L^{-2}$ screening does not yet play a role and controlling this phenomenon is, in fact, one of the accomplishments in this paper. On the other hand, [16] contains results about Gamma convergence, about the asymptotically uniform distribution of mass and energy, and about the droplet structure of minimizers.

The even more dilute situation where $\vartheta \sim L^{-3}$ was considered by Choksi and Peletier [4]. Then, the leading order $E\left(A^{*}\right) / A^{*}$ in (1.5) and (1.6) should be replaced by $E\left(\vartheta L^{3}\right) /\left(\vartheta L^{3}\right)$. In this situation our upper bound (1.5) is not applicable (at least not if $\vartheta L^{3}$ is too small), and our lower bound (1.6) is not tight. However, a simple variation of our arguments would also cover this regime. On the other hand, [4] also establishes a lower order correction to the energy and contains results about Gamma convergence.

It is conjectured that for small $\vartheta$ minimizers are given, at least in the bulk, by a periodic arrangement of nearly spherical sets. Our main result provides evidence for the latter prediction. If this conjecture is true, then, as the nucleon density $\vartheta$ tends to zero, the balls on the lattice should move infinitely far apart. Each one of the balls should therefore be asymptotically equal to an energy-per-volume minimizer of the full-space energy functional (1.1), and hence the energy per unit volume should be given to leading order by $\inf _{0<|\Omega|<\infty}|\Omega|^{-1} \mathcal{E}[\Omega]=\left(A^{*}\right)^{-1} E\left(A^{*}\right)$. This intuition guides us in the proof of the upper bound (1.5).

We emphasize that our result is valid independently of whether $\Omega^{*}$ is a ball or not. This is particularly relevant for the proof of the upper bound (1.5). When $\Omega^{*}$ is a ball, or more generally, when the dipole and quadrupole moments of $\Omega^{*}$ vanish, the proof of the upper bound (1.5) is straightforward following the above intuition. When the dipole and quadrupole moments of $\Omega^{*}$ do not vanish, we need to distort the lattice to achieve the required cancellation in the long-range behavior of the Coulomb potential.

The problem of proving periodicity of minimizers in this and other multidimensional minimization problems is a well-known and long standing open problem (crystallization conjecture). The strongest result about local order for the present problem was shown in the work [1] mentioned before. Remarkably, for the present problem it was proposed in the physics literature $[21,12]$ that there are phase transitions at $0<\vartheta_{c 1}<\vartheta_{c 2}<1 / 2<\vartheta_{c 3}=1-\vartheta_{c 2}<\vartheta_{c 4}=1-\vartheta_{c 1}<1$, where the dimensionality of the periodicity changes. For $0<\vartheta<\vartheta_{c 1}$, minimizers are expected to be sphere-shaped and arranged in a three-dimensional lattice, for $\vartheta_{c 1}<\vartheta<\vartheta_{c 2}$, minimizers are expected to be cylinder-shaped and arranged in a two-periodic lattice, and for $\vartheta_{c 2}<\vartheta<\vartheta_{c 3}$, minimizers are expected to be slab-shaped with respect to a one-dimensional lattice. For $\vartheta>1 / 2$ the situation reverses (since $\vartheta \mapsto 1-\vartheta$ corresponds to $\Omega \mapsto \mathbb{R}^{3} \backslash \Omega$ ), and one expects a transition to cylindrical holes and then to spherical holes. This phenomenon is sometimes referred to as "nuclear pasta phases." Numerically, one has $\vartheta_{c 1} \approx 0.20$ and $\vartheta_{c 2} \approx 0.35$ [20]. We refer the reader to a recent result [10] where the optimality of slab-like structures was rigorously established in a multidimensional lattice model which, similarly to the present model, contains an attractive short-range term competing with a repulsive long-range term; see also $[6,11]$.

In the literature the Coulomb kernel $|x-y|^{-1}$ in the energy (1.2) is often replaced by the Green's function of the Laplacian on $Q_{L}$ with some boundary condition. Apart 
from the "whole space" condition that we use, common choices are the periodic, the Dirichlet, and the Neumann boundary condition. That is, the energy functional under study is

$$
\mathcal{E}_{\#, L}[\Omega]:=\operatorname{Per}(\Omega)+\frac{1}{2} \int_{Q_{L}} \int_{Q_{L}}\left(1_{\Omega}(x)-\vartheta\right) G_{\#, L}(x, y)\left(1_{\Omega}(y)-\vartheta\right) \mathrm{d} x \mathrm{~d} y
$$

with the corresponding ground state energy

$$
E_{\#, L}(\vartheta):=\inf \left\{\mathcal{E}_{\#}[\Omega]: \Omega \subset Q_{L},|\Omega|=\vartheta L^{3}\right\}
$$

and thermodynamic limit

$$
e_{\#}(\vartheta):=\lim _{L \rightarrow \infty} \frac{E_{\#, L}(\vartheta)}{L^{3}} .
$$

Here, the symbol \# is a placeholder for the indices $P, D$, and $N$ denoting periodic, Dirichlet, and Neumann boundary conditions, respectively, and the corresponding Green's functions on $Q_{L}$ are denoted by $G_{P, L}, G_{D, L}$, and $G_{N, L}$, respectively. If we let, moreover, $G_{\infty, L}(x, y)=\frac{1}{4 \pi}|x-y|^{-1}$ be the whole space Green's function used above (up to rescaling) and allow for $\#=\infty$ in (1.9)-(1.11), we can state the following theorem.

Theorem 1.5 (Independence from boundary conditions). There are constants $\sigma^{*}>0$ and $C>0$ depending only on $\vartheta$ such that

$$
\left|\frac{E_{\#, L}(\theta)}{L^{3}}-\sigma^{*}\right| \leq \frac{C}{L}
$$

for every $L \geq C$ and every $\#=D, N, P, \infty$. In particular,

$$
e_{D}(\vartheta)=e_{N}(\vartheta)=e_{P}(\vartheta)=e_{\infty}(\vartheta)=\sigma^{*} .
$$

In the Neumann case, the existence of the thermodynamic limit, together with the sharp convergence rate of order $L^{-1}$, was shown in a remarkable paper by Alberti, Choksi, and Otto [1]. We use comparison arguments to extend their result to all the boundary conditions mentioned above; see section 5 . In particular, this shows that the left side in (1.7) is independent of the choice of boundary values.

The remainder of this paper consists of four sections. Section 2 deals with the upper bound (1.5) under the additional assumption that the energy-per-volume minimizer $\Omega^{*}$ is a ball. In section 3 we describe the necessary changes to remove this assumption. Section 4 deals with the lower bound (1.6). Section 5 is devoted to the proof of Theorem 1.5.

Notation. Since cubes with different sizes and centers will be a recurring tool in our analysis, it is convenient to introduce the following notation. For $r=\left(r_{1}, r_{2}, r_{3}\right) \in$ $\mathbb{R}^{3}$, we set

$$
|r|_{\infty}=\max \left\{\left|r_{i}\right|: i=1,2,3\right\}
$$

Then, for $r \in \mathbb{R}^{3}$ and $l>0$, we define

$$
Q_{l}(r):=\left\{x \in \mathbb{R}^{3}:|x-l r|_{\infty}<l / 2\right\}
$$

and $Q_{l}:=Q_{l}(0)$. Pay attention to the fact that by definition, $Q_{l}(r)$ is the cube of side length $l$ centered at the point $l r$-not at $r$ ! In other words, to obtain $Q_{l}(r)$, one first takes a cube of unit side length centered at $r$ and then dilates it by the factor $l$. We found this slightly unusual definition better suited for our purposes. 
2. Upper bound on the ground state energy if $\Omega^{*}$ is a ball. The purpose of this section and the next is to prove the first statement of Theorem 1.1, which we restate here for convenience.

Proposition 2.1 (Upper bound). There is a constant $C>0$ such that, if $\vartheta^{1 / 3} L \geq$ $C$ and $\vartheta \leq \frac{1}{2}$, we have

$$
\frac{E_{\vartheta, L}}{\vartheta L^{3}} \leq \frac{E\left(A^{*}\right)}{A^{*}}+C \vartheta^{1 / 3}+\frac{C}{\vartheta^{1 / 3} L} .
$$

In order to not obscure the simple underlying idea, throughout this section we make the additional assumption that the minimizer $\Omega^{*}$ of the whole space problem is a ball. As explained in the introduction, this is strongly conjectured to be the case. In section 3 we explain the technical modifications of the proof to treat the case of general $\Omega^{*}$.

Proof of Proposition 2.1 when $\Omega^{*}$ is a ball. We construct, for every pair $(\vartheta, L)$, a suitable competitor set $\Omega_{\vartheta, L}$ for $E_{\vartheta, L}$. The idea is to take $\Omega_{\vartheta, L}$ to be given by a cubic lattice arrangement on $Q_{L}$ of sets $\Omega^{*}$. The period length $l>0$ of the lattice will be chosen so that the requirement $\left|\Omega_{\vartheta, L}\right|=\vartheta L^{3}$ is fulfilled. Since we want each box of side length $l$ to contain one copy of $\Omega^{*}$, for the mass density to be equal to $\vartheta$, we need to require $\vartheta l^{3}=A^{*}$, or

$$
l=A^{* 1 / 3} \vartheta^{-1 / 3} .
$$

Let $\mathcal{C}_{\vartheta, L}:=\left\{r \in \mathbb{Z}^{3}: Q_{l}(r) \subset Q_{L}\right\}$ be the set of lattice points $r$ such that the cubes $Q_{l}(r)$ are fully contained in $Q_{L}$, and let $N_{\vartheta, L}:=\# \mathcal{C}_{\vartheta, L}$ denote the number of these cubes. We draw the reader's attention to our definition of cubes $Q_{l}(r)$ explained at the end of the introduction.

We now define the set $\Omega_{\vartheta, L}$ to be the following disjoint union:

$$
\Omega_{\vartheta, L}:=\bigcup_{r \in \mathcal{C}_{\vartheta, L}}\left(\lambda_{\vartheta, L} l r+\lambda_{\vartheta, L} \Omega^{*}\right)
$$

where the rescaling factor $\lambda_{\vartheta, L}$ is given by

$$
\lambda_{\vartheta, L}^{3}=\frac{\vartheta L^{3}}{A^{*} N_{\vartheta, L}} .
$$

We recall that we assume that $\Omega^{*}$ is a ball, which we assume to be centered at the origin. Moreover, we denote by $r_{*}$ its radius.

Note that the union in (2.2) is disjoint since $\vartheta \leq \frac{1}{2}$. (Indeed, we have $\frac{1}{2} l^{3} \geq \vartheta l^{3}=$ $A^{*}=\frac{4 \pi}{3} r_{*}^{3}$ and, hence, $l>2 r_{*}$.)

Informally, our construction of the competitor set $\Omega_{\vartheta, L}$ can thus be described as follows. We fill $Q_{L}$ with small boxes $Q_{l}(r)$ of side length $l$ as full as possible, place a copy of $\Omega^{*}$ in the middle of each box, and enlarge the whole configuration slightly by the factor $\lambda_{\vartheta, L}$.

The definition of $\lambda_{\vartheta, L}$ now ensures that the boxes $Q_{\lambda_{\vartheta, L} l}(r)$ cover $Q_{L}$ completely and that the mass constraint

$$
\left|\Omega_{\vartheta, L}\right|=N_{\vartheta, L} A^{*} \lambda_{\vartheta, L}^{3}=\vartheta L^{3}
$$

is fulfilled. Note also that with this choice, we even have local neutrality of $\Omega_{\vartheta, L}$ on every box $Q_{\lambda_{\vartheta, L} l}(r)$; i.e., for every $r \in \mathcal{C}_{\vartheta, L}$,

$$
\left|\Omega_{\vartheta, L} \cap Q_{\lambda_{\vartheta, L} l}(r)\right|=\lambda_{\vartheta, L}^{3} A^{*}=\vartheta \lambda_{\vartheta, L}^{3} l^{3}=\vartheta\left|Q_{\lambda_{\vartheta, L} l}(r)\right| .
$$


Since the number of boundary boxes is of order $\frac{L^{2}}{l^{2}}$, it is easy to see that $N_{\vartheta, L}$ satisfies the bounds

$$
\frac{L^{3}}{l^{3}} \geq N_{\vartheta, L} \geq \frac{L^{3}}{l^{3}}-C \frac{L^{2}}{l^{2}}
$$

for some $C>0$ independent of $\vartheta$ and $L$. From (2.3), we thus obtain the bound

$$
1 \leq \lambda_{\vartheta, L}^{3} \leq \frac{\vartheta L^{3}}{A^{*}\left(\frac{L^{3}}{l^{3}}-C \frac{L^{2}}{l^{2}}\right)}=\frac{\vartheta}{A^{*}\left(l^{-3}-C L^{-1} l^{-2}\right)}=\frac{1}{1-C \frac{l}{L}} \leq 1+C \frac{l}{L},
$$

and so, in particular, $\lim _{L \rightarrow \infty} \lambda_{\vartheta, L}=1$.

In many situations below, to estimate subleading terms, the crude bound

$$
1 \leq \lambda_{\vartheta, L} \leq 2
$$

is enough. It follows from (2.7) whenever $\frac{l}{L}=\frac{A^{* 1 / 3}}{\vartheta^{1 / 3} L} \leq C^{-1}$ for some universal $C>0$.

Our proof of the bound (2.1) consists in computing in three separate steps the self-energy, the near-field interaction energy, and the far-field interaction energy of the set $\Omega_{\vartheta, L}$. That is, we split

$$
\mathcal{E}_{\vartheta, L}\left[\Omega_{\vartheta, L}\right]=\mathcal{E}_{\vartheta, L}^{(\text {self })}+\mathcal{E}_{\vartheta, L}^{(\text {near })}+\mathcal{E}_{\vartheta, L}^{(\text {far })}
$$

by partitioning the double integral from the interaction term of $\mathcal{E}_{\vartheta, L}$. To simplify notation, we will write

$$
\tilde{l}=\lambda_{\vartheta, L} l
$$

in the rest of this proof. Hence, we define

$$
\mathcal{E}_{\vartheta, L}^{\text {(self) }}:=\operatorname{Per}\left(\Omega_{\vartheta, L}\right)+\sum_{r \in \mathcal{C}_{\vartheta, L}} \frac{1}{2} \int_{Q_{\tilde{l}}(r)} \int_{Q_{\tilde{l}}(r)}\left(1_{\Omega_{\vartheta, L}}(x)-\vartheta\right) \frac{1}{|x-y|}\left(1_{\Omega_{\vartheta, L}}(y)-\vartheta\right) \mathrm{d} x \mathrm{~d} y
$$

and

$$
\mathcal{E}_{\vartheta, L}^{(\text {near })}:=\sum_{(r, s) \in V_{\text {near }}} \frac{1}{2} \int_{Q_{\tilde{\tau}}(r)} \int_{Q_{\tilde{l}}(s)}\left(1_{\Omega_{\vartheta, L}}(x)-\vartheta\right) \frac{1}{|x-y|}\left(1_{\Omega_{\vartheta, L}}(y)-\vartheta\right) \mathrm{d} x \mathrm{~d} y
$$

where

$$
V_{\text {near }}:=V_{\vartheta, L}^{(\text {near })}:=\left\{(r, s) \in \mathcal{C}_{\vartheta, L} \times \mathcal{C}_{\vartheta, L} \text { and } 1 \leq|r-s|_{\infty} \leq M\right\} .
$$

Here, $M \in \mathbb{N}$ is a number fixed throughout our proof (let us say $M=10$ ).

Finally, we define

$$
\mathcal{E}_{\vartheta, L}^{(\mathrm{far})}:=\sum_{(r, s) \in V_{\mathrm{far}}} \frac{1}{2} \int_{Q_{\tilde{l}}(r)} \int_{Q_{\tilde{l}}(s)}\left(1_{\Omega_{\vartheta}, L}(x)-\vartheta\right) \frac{1}{|x-y|}\left(1_{\Omega_{\vartheta, L}}(y)-\vartheta\right) \mathrm{d} x \mathrm{~d} y
$$

where

$$
V_{\text {far }}:=V_{\vartheta, L}^{(\mathrm{far})}:=\left\{(r, s) \in \mathcal{C}_{\vartheta, L} \times \mathcal{C}_{\vartheta, L} \text { and }|r-s|_{\infty}>M\right\}
$$


Step 1: Self-energy. Similarly to $\tilde{l}$, we write

$$
\tilde{\Omega}^{*}=\lambda_{\vartheta, L} \Omega^{*}
$$

here and in the rest of this proof. Since $\Omega_{\vartheta, L}$ consists of $N_{\vartheta, L}$ disjoint copies of $\tilde{\Omega}^{*}$, we have

$$
\frac{\mathcal{E}_{\vartheta, L}^{(\text {self })}}{\vartheta L^{3}} \leq \frac{N_{\vartheta, L}}{\vartheta L^{3}}\left(\operatorname{Per}\left(\tilde{\Omega}^{*}\right)+\frac{1}{2} \int_{\tilde{\Omega}^{*}} \int_{\tilde{\Omega}^{*}} \frac{\mathrm{d} x \mathrm{~d} y}{|x-y|}+\frac{\vartheta^{2}}{2} \int_{Q_{\tilde{l}}} \int_{Q_{\tilde{l}}} \frac{\mathrm{d} x \mathrm{~d} y}{|x-y|}\right) .
$$

Since $\frac{N_{\vartheta, L}}{\vartheta L^{3}}=\frac{1}{A^{*} \lambda_{\vartheta, L}^{3}}$ by $(2.4)$, we have

$$
\begin{aligned}
\frac{\mathcal{E}_{\vartheta, L}^{(\text {self })}}{\vartheta L^{3}} & \leq \frac{1}{A^{*}}\left(\lambda_{\vartheta, L}^{-1} \operatorname{Per}\left(\Omega^{*}\right)+\frac{\lambda_{\vartheta, L}^{2}}{2} \int_{\Omega^{*}} \int_{\Omega^{*}} \frac{\mathrm{d} x \mathrm{~d} y}{|x-y|} \mathrm{d} x \mathrm{~d} y+C \vartheta^{2} \tilde{l}^{5}\right) \\
& \leq \frac{E\left(A^{*}\right)}{A^{*}}+\frac{E\left(A^{*}\right)}{A^{*}}\left(\lambda_{\vartheta, L}^{2}-1\right)+C \vartheta^{2} l^{5} \\
& \leq \frac{E\left(A^{*}\right)}{A^{*}}+\frac{C}{\vartheta^{1 / 3} L}+C \vartheta^{1 / 3},
\end{aligned}
$$

where we used the bound $\lambda_{\vartheta, L}^{2}-1 \leq C\left(\lambda_{\vartheta, L}^{3}-1\right) \leq C \frac{l}{L}=C \frac{1}{\vartheta^{1 / 3} L}$ from (2.7) for the last inequality. Moreover, recall that $l \leq \tilde{l}=\lambda_{\vartheta, L} l \leq 2 l$ from (2.8).

Step 2: Near-field interaction. Due to the periodicity of $\Omega_{\vartheta, L}$, we have

$$
\begin{aligned}
\frac{\mathcal{E}_{\vartheta, L}^{(\text {near })}}{\vartheta L^{3}} & =\frac{1}{\vartheta L^{3}} \sum_{(r, s) \in V_{\text {near }}} \frac{1}{2} \int_{Q_{\tilde{l}}(r)} \int_{Q_{\tilde{l}}(s)}\left(1_{\Omega_{\vartheta}, L}(x)-\vartheta\right) \frac{1}{|x-y|}\left(1_{\Omega_{\vartheta, L}}(y)-\vartheta\right) \mathrm{d} x \mathrm{~d} y \\
& =\frac{1}{\vartheta L^{3}} \sum_{(r, s) \in V_{\text {near }}} \frac{1}{2} \int_{Q_{\tilde{l}}} \int_{Q_{\tilde{l}}}\left(1_{\tilde{\Omega}^{*}}(x)-\vartheta\right) \frac{1}{|r \tilde{l}+x-s \tilde{l}-y|}\left(1_{\tilde{\Omega}^{*}}(y)-\vartheta\right) \mathrm{d} x \mathrm{~d} y \\
(2.17) & \leq \frac{1}{\vartheta L^{3}} \sum_{(r, s) \in V_{\text {near }}} \frac{1}{2}\left(\int_{\tilde{\Omega}^{*}} \int_{\tilde{\Omega}^{*}} \frac{1}{|r \tilde{l}+x-s \tilde{l}-y|} \mathrm{d} x \mathrm{~d} y+\vartheta^{2} \int_{Q_{\tilde{l}}} \mathrm{~d} x \int_{Q_{\tilde{l}}} \frac{1}{|y|} \mathrm{d} y\right),
\end{aligned}
$$

where we used the fact that the integral over the symmetric-decreasing function $1 /|\cdot|$ is largest on the cube centered at 0 . This follows from the observation that three Steiner symmetrizations with respect to the coordinate directions $e_{1}, e_{2}, e_{3}$ transform any cube $Q_{l}(\mu)$ into the centered cube $Q_{l}(0)$. Furthermore, for every $r \neq s$ and $x, y \in \tilde{\Omega}^{*}$ we have

$$
|r \tilde{l}+x-s \tilde{l}-y| \geq|r-s| \tilde{l}-|x-y| \geq \lambda_{\vartheta, L}\left(l-\operatorname{diam}\left(\Omega^{*}\right)\right) \geq \tilde{l} / C .
$$

Here, we used $\frac{1}{2} l^{3} \geq \vartheta l^{3}=A^{*}=\frac{4 \pi}{3} r_{*}^{3}$, which implies $l-2 r_{*} \geq l / C$. Hence, the right-hand side of (2.17) is bounded from above by

$$
\frac{1}{\vartheta L^{3}} \sum_{r \in \mathcal{C}_{\vartheta, L}} M^{3}\left(\frac{C}{\tilde{l}}+C \vartheta^{2} \tilde{l}^{5}\right) \leq C \frac{L^{3} \tilde{l}^{-3}}{\vartheta L^{3}}\left\{\tilde{l}^{-1}+\vartheta^{2} \tilde{l}^{5}\right\} \leq C \vartheta^{1 / 3},
$$

where we used the bound (2.6). For the last inequality, recall the choice $\vartheta l^{3}=\left|\Omega^{*}\right|=$ $A^{*}$ and the bound $1 \leq \lambda_{\vartheta, L} \leq 2$ from (2.8). 
Step 3: Far-field interaction. Due to the periodicity of $\Omega_{\vartheta, L}$, we have

$$
\begin{aligned}
\mathcal{E}_{\vartheta, L}^{(\mathrm{far})} & =\sum_{(r, s) \in V_{\mathrm{far}}} \frac{1}{2} \int_{Q_{\tilde{l}}(r)} \int_{Q_{\tilde{l}}(s)}\left(1_{\Omega_{\vartheta, L}}(x)-\vartheta\right) \frac{1}{|x-y|}\left(1_{\Omega_{\vartheta, L}}(y)-\vartheta\right) \mathrm{d} x \mathrm{~d} y \\
& =\sum_{(r, s) \in V_{\mathrm{far}}} \frac{1}{2} \int_{Q_{\tilde{l}}} \int_{Q_{\tilde{l}}}\left(1_{\tilde{\Omega}^{*}}(x)-\vartheta\right) \frac{1}{|r \tilde{l}+x-s \tilde{l}-y|}\left(1_{\tilde{\Omega}^{*}}(y)-\vartheta\right) \mathrm{d} x \mathrm{~d} y .
\end{aligned}
$$

We now use the Taylor expansion

$$
\frac{1}{|a-b|}=\frac{1}{|a|}+\frac{a \cdot b}{|a|^{3}}+\frac{1}{2} \frac{3(a \cdot b)^{2}-a^{2} b^{2}}{|a|^{5}}+\mathcal{O}\left(\frac{|b|^{3}}{|a|^{4}}\right)
$$

valid for $a, b \in \mathbb{R}^{3}$ with $|a| \geq 4|b|$, and choose $a=(r-s) \tilde{l}+x$ and $b=y$.

By our assumption that $\Omega^{*}=B\left(0, r_{*}\right)$, the monopole, the dipole, and the quadrupole moments of $1_{\Omega^{*}}-\vartheta 1_{Q_{\tilde{i}}}$ vanish.

That is, for all $a \in \mathbb{R}^{3} \backslash\{0\}$, we have the equation

$$
\int_{\mathbb{R}^{3}}\left(1_{\tilde{\Omega}^{*}}(y)-\vartheta 1_{Q_{\tilde{l}}}(y)\right)\left(\frac{1}{|a|}+\frac{a \cdot y}{|a|^{3}}+\frac{1}{2} \frac{3(a \cdot y)^{2}-a^{2} y^{2}}{|a|^{5}}\right) \mathrm{d} y=0 .
$$

This follows from our neutrality condition (2.5) and the symmetries of a ball and a cube centered at 0 . More precisely, the function $1_{\tilde{\Omega}^{*}}(y)-\vartheta 1_{Q_{\tilde{l}}}(y)$ is invariant under the reflection of one coordinate $y_{i} \mapsto-y_{i}$ as well as under the exchange of two coordinates $y_{i}$ and $y_{j}$.

These symmetries cause the dipole, respectively, the quadrupole, moment to vanish. We stress that this is one of only two places where the additional assumption $\Omega^{*}=B\left(0, r_{*}\right)$ enters into our proof. The other one is the fact that $\Omega^{*}=B\left(0, r_{*}\right) \subset Q_{l}$ for $\vartheta \leq \frac{1}{2}$ if $\vartheta l^{3}=\left|\Omega^{*}\right|$. We again refer the reader to section 3 for the necessary modifications to obtain an equation similar to (2.21) in the absence of the assumption $\Omega^{*}=B\left(0, r_{*}\right)$.

By (2.21), if we plug in the expansion $(2.20)$ and set $a=(r-s) \tilde{l}+x$ and $b=y$, then (2.19) is bounded from above by

$$
\begin{aligned}
& C \sum_{(r, s) \in V_{\mathrm{far}}} \int_{Q_{\tilde{l}}} \int_{Q_{\tilde{\imath}}}\left|1_{\tilde{\Omega}^{*}}(x)-\vartheta\right| \frac{|y|^{3}}{|r \tilde{l}-s \tilde{l}+x|^{4}}\left|1_{\tilde{\Omega}^{*}}(y)-\vartheta\right| \mathrm{d} x \mathrm{~d} y \\
\leq & C \sum_{(r, s) \in V_{\mathrm{far}}} \int_{Q_{\tilde{l}}} \frac{1_{\tilde{\Omega}^{*}}(x)+\vartheta}{|(r-s) \tilde{l}+x|^{4}} \mathrm{~d} x \int_{Q_{\tilde{\imath}}}\left(1_{\tilde{\Omega}^{*}}(y)+\vartheta\right)|y|^{3} \mathrm{~d} y .
\end{aligned}
$$

For $x \in Q_{\tilde{l}}$ we have $|x| \leq \sqrt{3} \tilde{l} / 2$. Since $|r-s|>M=10$, it follows that $|(r-s) \tilde{l}+x| \geq$ $\tilde{l}|r-s|-|x| \geq \frac{1}{2} \tilde{l}|r-s|$. The right-hand side of inequality (2.22) can thus be estimated from above by

$$
C \sum_{(r, s) \in V_{\text {far }}} \frac{2^{4}}{\tilde{l}^{4}|r-s|^{4}} \int_{Q_{\tilde{\imath}}}\left(1_{\tilde{\Omega}^{*}}(x)+\vartheta\right) \mathrm{d} x\left(\int_{\tilde{\Omega}^{*}}|y|^{3} \mathrm{~d} y+\vartheta \int_{Q_{\tilde{l}}}|y|^{3} \mathrm{~d} y\right)
$$

$$
\leq \frac{C}{\tilde{l}^{4}} \sum_{(r, s) \in V_{\text {far }}} \frac{1}{|r-s|^{4}}\left(1+\vartheta \tilde{l}^{3}\right)\left(1+\vartheta \tilde{l}^{6}\right) \leq C l^{-1}\left(1+\vartheta l^{3}\right)\left(l^{-3}+\vartheta l^{3}\right) \sum_{(r, s) \in V_{\text {far }}} \frac{1}{|r-s|^{4}}
$$


where we again used $1 \leq \lambda_{\vartheta, L} \leq 2$, and, hence, $l \leq \tilde{l} \leq 2 l$, from (2.8).

Since $\vartheta l^{3}=A^{*}$, it remains to evaluate the last sum over the set $V_{\mathrm{far}}$. Recalling the bound on the number of boxes $N_{\vartheta, L} \leq \frac{L^{3}}{l^{3}}$, we have

$$
\sum_{(r, s) \in V_{\mathrm{far}}} \frac{1}{|r-s|^{4}} \leq \sum_{r \in \mathcal{C}_{\vartheta, L}} \sum_{\substack{s \in \mathbb{Z}^{3} \\ s \neq r}} \frac{1}{|r-s|^{4}} \leq \frac{L^{3}}{l^{3}} \sum_{s \in \mathbb{Z}^{3} \backslash\{0\}} \frac{1}{|s|^{4}}=C \vartheta L^{3} .
$$

Putting together (2.19), (2.23), and (2.24) and using $\vartheta l^{3}=A^{*}$, we obtain

$$
\frac{1}{\vartheta L^{3}} \mathcal{E}_{\vartheta, L}^{(\mathrm{far})} \leq C l^{-1}=C \vartheta^{1 / 3}
$$

Step 4: Conclusion. Inserting the bounds proved in steps 1-3 back into (2.9), we obtain

$$
\frac{\mathcal{E}_{\vartheta, L}\left[\Omega_{\vartheta, L}\right]}{\vartheta L^{3}}=\frac{\mathcal{E}_{\vartheta, L}^{(\text {self })}+\mathcal{E}_{\vartheta, L}^{(\text {near })}+\mathcal{E}_{\vartheta, L}^{(\mathrm{far})}}{\vartheta L^{3}} \leq \frac{E\left(A^{*}\right)}{A^{*}}+C \vartheta^{1 / 3}+C \frac{1}{\vartheta^{1 / 3} L} .
$$

The proof of Proposition 2.1 is therefore complete.

3. Upper bound on the ground state energy in the general case. We give here the necessary modifications to obtain the upper bound from Theorem 1.1 if one does not make any symmetry assumption on the energy-per-volume minimizer $\Omega^{*}$.

In case $\vartheta>1 / C$, inequality (1.5) is equivalent to the bound

$$
\frac{E_{\vartheta, L}}{\vartheta L^{3}} \leq C^{\prime} .
$$

To show this, we do not need to use minimizers in the construction of our test set $\Omega_{\vartheta, L}$. It suffices to consider balls of any fixed radius $r_{*}>0$ arranged on a lattice just as it is done in the proof of Proposition 2.1. Henceforth we may therefore assume

$$
\vartheta \leq \frac{1}{C}
$$

The proof strategy of Theorem 1.1 in the absence of symmetry of $\Omega^{*}$ is identical to the one of the upper bound in section 2 . One constructs a competitor set made from energy-per-volume minimizers $\Omega^{*}$ arranged on a lattice. The difficulty one faces is that in proving the error bound on the far-field interaction term, one cannot invoke the symmetry of $\Omega^{*}$ to prove that the monopole, dipole, and quadrupole moments vanish as in (2.21).

We resolve this difficulty by fine-tuning the parameters of our lattice. More precisely, we show that the analogue of $(2.21)$ can still be achieved by considering a suitably translated and rotated copy of $\Omega^{*}$, arranged on a slightly distorted lattice.

Notation. To deal with cuboids instead of cubes, it is necessary to introduce some appropriate notation. For $r \in \mathbb{R}^{3}$ and $\boldsymbol{l} \in \mathbb{R}^{3}$, we define

$$
Q_{l}(r):=\left\{x \in \mathbb{R}^{3}:\left|x_{i}-l_{i} r_{i}\right|<l_{i} / 2 \text { for } i \in\{1,2,3\}\right\}
$$

and $Q_{l}:=Q_{l}(0)$. Once again, pay attention to the fact that by definition, $Q_{l}(r)$ is the cuboid of side lengths $\boldsymbol{l}$ centered at the point with coordinates $l_{i} r_{i}$-not centered 
at $r$ ! This is because we intend to cover $Q_{L}$ with many copies of the cuboid of side lengths $\boldsymbol{l}$. Then, the parameter $r \in \mathbb{Z}^{3}$ simply counts those cuboids in each direction.

More generally, given $\boldsymbol{\lambda}=\left(\lambda_{1}, \lambda_{2}, \lambda_{3}\right) \in \mathbb{R}^{3}$ and $\Omega \subset \mathbb{R}^{3}$, we define the "inhomogeneous dilation" by $\boldsymbol{\lambda}$ of the set $\Omega$ to be $\boldsymbol{\lambda} \Omega:=\left\{\left(\lambda_{1} x_{1}, \lambda_{2} x_{2}, \lambda_{3} x_{3}\right): x \in \Omega\right\}$. Observe that with these definitions, one has $\lambda Q_{L}=Q_{L \lambda}$. We shall use both notations according to convenience.

Furthermore, for $\Omega \in \lambda Q_{L}$, we set

$$
\mathcal{E}_{\vartheta, L, \boldsymbol{\lambda}}[\Omega]=\operatorname{Per}(\Omega)+\frac{1}{2} \int_{\boldsymbol{\lambda} Q_{L}} \int_{\boldsymbol{\lambda} Q_{L}}\left(1_{\Omega}(x)-\vartheta\right)|x-y|^{-1}\left(1_{\Omega}(y)-\vartheta\right) \mathrm{d} x \mathrm{~d} y
$$

and define the corresponding ground state energy by

$$
E_{\vartheta, L, \boldsymbol{\lambda}}=\inf \left\{\mathcal{E}_{\vartheta, L, \boldsymbol{\lambda}}[\Omega]: \Omega \subset \boldsymbol{\lambda} Q_{L},|\Omega|=\vartheta\left|\boldsymbol{\lambda} Q_{L}\right|\right\} .
$$

With this notation at hand, we can prove the following two key lemmas.

LEMma 3.1 (Vanishing multipole moments). Let $\Omega \subset \mathbb{R}^{3}$ be a bounded set. Assume that two numbers $l_{0}>0$ and $\vartheta \in(0,1]$ are given such that $|\Omega|=\vartheta l_{0}^{3}$. If $\eta_{0}:=\frac{l_{0}}{\operatorname{diam}(\Omega)}$ is larger than a universal constant, then there is an orthogonal matrix $U \in \mathbb{R}^{3 \times 3}$, a translation vector $y \in \mathbb{R}^{3}$, and a scaling vector $\boldsymbol{l}=\boldsymbol{\lambda} l_{0}$ such that the set $\Omega_{0}:=U(\Omega+y)$ is contained in $Q_{l}$ and satisfies

$$
\begin{aligned}
0 & =\int_{\mathbb{R}^{3}}\left(1_{\Omega_{0}}(x)-\vartheta 1_{Q_{l}}(x)\right) \mathrm{d} x=\int_{\mathbb{R}^{3}} x_{i}\left(1_{\Omega_{0}}(x)-\vartheta 1_{Q_{l}}(x)\right) \mathrm{d} x \\
& =\int_{\mathbb{R}^{3}}\left(3 x_{i} x_{j}-\delta_{i j}|x|^{2}\right)\left(1_{\Omega_{0}}(x)-\vartheta 1_{Q_{l}}(x)\right) \mathrm{d} x
\end{aligned}
$$

for all $i, j \in\{1,2,3\}$. Furthermore, the scaling parameters $\boldsymbol{\lambda}=\left(\lambda_{1}, \lambda_{2}, \lambda_{3}\right)$ satisfy

$$
\lambda_{1} \lambda_{2} \lambda_{3}=1 \quad \text { and } \quad\left|\lambda_{i}-1\right| \leq C \eta_{0}^{-2} \quad(i=1,2,3)
$$

for a universal constant $C>0$.

Remark 3.2. We point out that since the proof below does not use the special form of $1_{\Omega}$ as an indicator function, the statement of Lemma 3.1 remains true if one replaces $1_{\Omega}$ by an arbitrary charge distribution $\rho \geq 0, \rho \in L^{1}\left(\mathbb{R}^{3}\right)$, with compact support.

Proof of Lemma 3.1. Let $\Omega \subset \mathbb{R}^{3}$ satisfy $|\Omega|=\vartheta l_{0}^{3}$. We first observe that since rotations and translations do not change the volume $|\Omega|=\vartheta l_{0}^{3}$, we always have

$$
0=\int_{\mathbb{R}^{3}}\left(1_{U(\Omega+y)}(x)-\vartheta 1_{Q_{l}}(x)\right) \mathrm{d} x
$$

as long as the constraint $\lambda_{1} \lambda_{2} \lambda_{3}=1$ is satisfied, which implies $\left|Q_{l}\right|=l_{0}^{3}$.

Next, we claim that up to replacing $\Omega$ by its translate $\Omega+y$ for a suitable vector $y \in \mathbb{R}^{3}$, we may achieve that

$$
0=\int_{\mathbb{R}^{3}} x_{i}\left(1_{\Omega}(x)-\vartheta 1_{Q_{l}}\right) \mathrm{d} x \quad \text { for all } i=1,2,3,
$$

for every $\boldsymbol{l} \in \mathbb{R}^{3}$. Indeed, the cube $Q_{l}$ is symmetric with respect to the coordinate planes, and thus

$$
0=\vartheta \int_{\mathbb{R}^{3}} x_{i} 1_{Q_{l}}(x) \mathrm{d} x .
$$


Moreover, for $y \in \mathbb{R}^{3}$ one has

$$
\int_{\mathbb{R}^{3}} 1_{\Omega+y}(x) x_{i} \mathrm{~d} x=\int_{\mathbb{R}^{3}} 1_{\Omega}(x)\left(x_{i}+y_{i}\right) \mathrm{d} x=\int_{\Omega} x_{i} \mathrm{~d} x+y_{i}|\Omega| .
$$

Hence it suffices to set $y_{i}=-\frac{1}{\Omega \mid} \int_{\Omega} x_{i} \mathrm{~d} x$. We continue for simplicity to denote the translated version $\Omega+y$ which satisfies (3.2) by $\Omega$. Note also that if $\Omega$ satisfies (3.2), then so does $U \Omega$ for any invertible matrix $U \in \mathbb{R}^{3 \times 3}$.

It remains to ensure the quadrupole moment vanishes by introducing appropriate $U \in \mathbb{R}^{3 \times 3}$ and $\boldsymbol{l}=\boldsymbol{\lambda} l_{0} \in \mathbb{R}^{3}$. Since the quadrupole moment of $\Omega$,

$$
P=\left(P_{i j}\right)_{i, j=1,2,3} \quad \text { with } \quad P_{i j}:=\int_{\Omega}\left(3 x_{i} x_{j}-\delta_{i j}|x|^{2}\right) \mathrm{d} x,
$$

is a traceless symmetric $3 \times 3$ matrix with real entries, there is an orthogonal matrix $U \in \mathbb{R}^{3 \times 3}$ and numbers $a, b \in \mathbb{R}$ such that

$(3.3)$

$$
\left[\begin{array}{ccc}
a & 0 & 0 \\
0 & b & 0 \\
0 & 0 & -a-b
\end{array}\right]=U P U^{T}=\int_{\Omega}\left(3(U x)_{i}(U x)_{j}-\delta_{i j}|U x|^{2}\right) \mathrm{d} x=\int_{U \Omega}\left(3 x_{i} x_{j}-\delta_{i j}|x|^{2}\right) \mathrm{d} x .
$$

That is, up to replacing $\Omega$ by its rotated version $U \Omega=: \Omega_{0}$, whose monopole and dipole moments still vanish by the remarks made above, we can assume that its quadrupole moment is diagonal.

To make the quadrupole moment of $\left(1_{\Omega_{0}}-\vartheta 1_{Q_{l}}\right)$ vanish, we thus need to find a cuboid $Q_{l}$ of volume $\left|Q_{l}\right|=l_{0}^{3}$ which contains $\Omega_{0}$ and satisfies

$$
\begin{gathered}
\vartheta \int_{Q_{l}}\left(3 x_{1}^{2}-|x|^{2}\right) \mathrm{d} x=a, \\
\vartheta \int_{Q_{l}}\left(3 x_{2}^{2}-|x|^{2}\right) \mathrm{d} x=b .
\end{gathered}
$$

Setting $l_{1}=\lambda_{1} l_{0}, l_{2}=\lambda_{2} l_{0}$, and $l_{3}=\lambda_{3} l_{0}=\frac{l_{0}}{\lambda_{1} \lambda_{2}}$ (by the volume constraint), and then by rescaling and using the relation $|\Omega|=\vartheta l_{0}^{3}$, we find that the system (3.4) is equivalent to

$$
\begin{aligned}
2 \lambda_{1}^{2}-\lambda_{2}^{2}-\frac{1}{\lambda_{1}^{2} \lambda_{2}^{2}} & =\frac{12}{|\Omega| l_{0}^{2}} a, \\
-\lambda_{1}^{2}+2 \lambda_{2}^{2}-\frac{1}{\lambda_{1}^{2} \lambda_{2}^{2}} & =\frac{12}{|\Omega| l_{0}^{2}} b .
\end{aligned}
$$

By adding these two equations, respectively, subtracting them, we obtain the equations

$$
\begin{aligned}
\lambda_{1}^{2}+\lambda_{2}^{2}-\frac{2}{\lambda_{1}^{2} \lambda_{2}^{2}} & =\frac{12(a+b)}{|\Omega| l_{0}^{2}}=: 2 c_{1}, \\
\lambda_{1}^{2}-\lambda_{2}^{2} & =\frac{4(a-b)}{|\Omega| l_{0}^{2}}=: 2 c_{2} .
\end{aligned}
$$


Inserting the second equation of (3.6) into the first one and changing to the center of mass coordinate $X=\left(\lambda_{1}^{2}+\lambda_{2}^{2}\right) / 2$, we get the equation

$$
X-\frac{1}{X^{2}-c_{2}^{2}}=c_{1}
$$

which is equivalent to the cubic equation

$$
p(X):=X^{3}-c_{1} X^{2}-c_{2}^{2} X-1+c_{1} c_{2}^{2}=0 .
$$

It can be seen from (3.3) that $|a+b| \leq 8 \operatorname{diam}\left(\Omega_{0}\right)^{2}\left|\Omega_{0}\right|$. Therefore, the definition (3.6) of the $c_{i}$ implies that $\left|c_{i}\right|<48 \operatorname{diam}\left(\Omega_{0}\right)^{2} l_{0}^{-2}$. Hence, if $\eta_{0}=\frac{l_{0}}{\operatorname{diam}\left(\Omega_{0}\right)}$ is large enough, the polynomial $p$ will be very close to $X^{3}-1$. Since $X^{3}-1$ has exactly one complex zero close to 1 (namely 1), we can apply Rouché's theorem in a ball of radius $\sim \eta_{0}^{-2}$ around 1 . Hence there exists exactly one complex zero $X_{0}$ of $p$ with $\left|X_{0}-1\right| \leq C \eta_{0}^{-2}$. Since the coefficients of $p$ are real, uniqueness of the zero implies that $X_{0}$ is in fact real.

We therefore get solutions $\lambda_{1}, \lambda_{2}>0$ of (3.6) which satisfy

$$
\begin{aligned}
& \left|\lambda_{1}-1\right|=\frac{\left|\lambda_{1}^{2}-1\right|}{\lambda_{1}+1}=\frac{\left|X_{0}+c_{2}-1\right|}{\lambda_{1}+1} \leq C \eta_{0}^{-2}, \\
& \left|\lambda_{2}-1\right|=\frac{\left|\lambda_{1}^{2}-1\right|}{\lambda_{1}+1}=\frac{\left|X_{0}-c_{2}-1\right|}{\lambda_{1}+1} \leq C \eta_{0}^{-2} .
\end{aligned}
$$

Note that $\lambda_{3}=1 /\left(\lambda_{1} \lambda_{2}\right)$ also satisfies $\left|\lambda_{3}-1\right| \leq C \eta_{0}^{-2}$. Moreover, the fact that $\int_{\Omega_{0}} x_{i} \mathrm{~d} x=0$ implies easily that $\Omega_{0} \subset Q_{l_{0}}$ for every $l_{0} \geq 2 \operatorname{diam}(\Omega)$. This completes the proof of Lemma 3.1.

Our next lemma shows that for $\boldsymbol{\lambda}$ close to $(1,1,1)$, we can replace the ground state energy of $Q_{L}$ by that of the cuboid $Q_{\boldsymbol{\lambda} L}$ with only a small error.

Lemma 3.3 (Approximating $E_{\vartheta, L}$ by a cuboid $E_{\vartheta, L, \boldsymbol{\lambda}}$ ). Suppose that $\boldsymbol{\lambda}=$ $\left(\lambda_{1}, \lambda_{2}, \lambda_{3}\right)$ is such that $\lambda_{1} \lambda_{2} \lambda_{3}=1$, and assume that $\lambda_{i} \in[1-\delta, 1+\delta]$ for $i=1,2,3$, for some $\delta \in[0,1]$. Then we have

$$
\mathcal{E}_{\vartheta, L}[\Omega] \leq(1+C \delta) \mathcal{E}_{\vartheta, L, \boldsymbol{\lambda}}[\boldsymbol{\lambda} \Omega] \quad \text { for all } \Omega \subset Q_{L},
$$

where $C>0$ is a universal constant independent of $\delta, \vartheta, L$, and $\Omega$. In particular, this implies

$$
E_{\vartheta, L} \leq(1+C \delta) E_{\vartheta, L, \lambda} .
$$

Proof. Let $\Omega \subset Q_{L}$ be arbitrary and consider, for $\boldsymbol{\lambda}$ as in the statement, the set $\lambda \Omega$. Note that since $\lambda_{1} \lambda_{2} \lambda_{3}=1$, we have $|\lambda \Omega|=|\Omega|$ and $\left|\lambda Q_{L}\right|=\left|Q_{L}\right|$.

To prove (3.9), we consider the perimeter and Coulomb terms separately. Let us assume for definiteness in the following that $\lambda_{1} \leq \lambda_{2} \leq \lambda_{3}$. First, recall the definition

$$
\operatorname{Per}(\Omega)=\sup \left\{\int_{\Omega} \operatorname{div} \varphi(x) \mathrm{d} x: \varphi \in C_{c}^{1}\left(\mathbb{R}^{3}, \mathbb{R}^{3}\right),\|\varphi\|_{\infty} \leq 1\right\} .
$$

For any $\varphi$ as in (3.11) and $\boldsymbol{\lambda} \in \mathbb{R}^{3}$ with $\lambda_{1} \lambda_{2} \lambda_{3}=1$, define the vector field $\varphi_{\boldsymbol{\lambda}} \in$ $C_{c}^{1}\left(\mathbb{R}^{3}, \mathbb{R}^{3}\right)$ by setting its $i$ th component to be $\varphi_{\boldsymbol{\lambda}, i}(x)=\lambda_{i} \varphi_{i}\left(\lambda_{1}^{-1} x_{1}, \lambda_{2}^{-1} x_{2}, \lambda_{3}^{-1} x_{3}\right)$. One easily checks that

$$
\int_{\Omega} \operatorname{div} \varphi(x) \mathrm{d} x=\int_{\boldsymbol{\lambda} \Omega} \operatorname{div} \varphi_{\boldsymbol{\lambda}}(x) \mathrm{d} x=\left\|\varphi_{\boldsymbol{\lambda}}\right\|_{\infty} \int_{\boldsymbol{\lambda} \Omega} \operatorname{div} \frac{\varphi_{\boldsymbol{\lambda}}(x)}{\left\|\varphi_{\boldsymbol{\lambda}}\right\|_{\infty}} \mathrm{d} x .
$$


Moreover, we estimate

$$
\left\|\varphi_{\boldsymbol{\lambda}}\right\|_{\infty}^{2}=\sup _{x \in \mathbb{R}^{3}} \sum_{i=1}^{3} \lambda_{i}^{2} \varphi_{i}^{2}\left(\lambda_{1}^{-1} x_{1}, \lambda_{2}^{-1} x_{2}, \lambda_{3}^{-1} x_{3}\right) \leq \lambda_{3}^{2}\|\varphi\|_{\infty}^{2} \leq 1+C \delta .
$$

In view of the definition (3.11) of the perimeter, we can take the sup over all $\varphi \in$ $C_{c}^{1}\left(\mathbb{R}^{3}, \mathbb{R}^{3}\right)$ with $\|\varphi\|_{\infty} \leq 1$ to obtain

$$
\operatorname{Per}(\Omega)=\sup _{\varphi} \int_{\Omega} \operatorname{div} \varphi(x) \mathrm{d} x=\sup _{\varphi}\left\|\varphi_{\boldsymbol{\lambda}}\right\|_{\infty} \int_{\boldsymbol{\lambda} \Omega} \operatorname{div} \frac{\varphi_{\boldsymbol{\lambda}}(x)}{\left\|\varphi_{\boldsymbol{\lambda}}\right\|_{\infty}} \mathrm{d} x \leq(1+C \delta) \operatorname{Per}(\boldsymbol{\lambda} \Omega),
$$

where we have used (3.13) for the last inequality.

To estimate the Coulomb term, it is convenient to pass to the Fourier representation. Set $f(x):=1_{\Omega}(x)-\vartheta 1_{Q_{L}}(x)$; then

$$
1_{\boldsymbol{\lambda} \Omega}(x)-\vartheta 1_{\boldsymbol{\lambda} Q_{L}}(x)=f\left(\lambda_{1}^{-1} x_{1}, \lambda_{2}^{-1} x_{2}, \lambda_{3}^{-1} x_{3}\right)=: f_{\boldsymbol{\lambda}}(x),
$$

and one easily computes that $\mathcal{F} f_{\boldsymbol{\lambda}}(p)=\mathcal{F} f\left(\lambda_{1} p_{1}, \lambda_{2} p_{2}, \lambda_{3} p_{3}\right)$. Therefore, we have $(3.15)$

$$
\begin{aligned}
& \frac{1}{4 \pi} \int_{Q_{L}} \int_{Q_{L}} \frac{\left(1_{\Omega}(x)-\vartheta\right)\left(1_{\Omega}(y)-\vartheta\right)}{|x-y|} \mathrm{d} x \mathrm{~d} y=\int_{\mathbb{R}^{3}} \frac{|\mathcal{F} f(p)|^{2}}{p^{2}} \mathrm{~d} p=\int_{\mathbb{R}^{3}} \frac{\left|\mathcal{F} f_{\boldsymbol{\lambda}}(p)\right|^{2}}{\sum_{i=1}^{3} \lambda_{i}^{2} p_{i}^{2}} \mathrm{~d} p \\
& \leq \lambda_{1}^{-2} \int_{\mathbb{R}^{3}} \frac{\left|\mathcal{F} f_{\boldsymbol{\lambda}}(p)\right|^{2}}{p^{2}} \mathrm{~d} p \leq(1+C \delta) \frac{1}{4 \pi} \int_{\boldsymbol{\lambda} Q_{L}} \int_{\boldsymbol{\lambda} Q_{L}} \frac{\left(1_{\boldsymbol{\lambda} \Omega}(x)-\vartheta\right)\left(1_{\boldsymbol{\lambda} \Omega}(y)-\vartheta\right)}{|x-y|} \mathrm{d} x \mathrm{~d} y .
\end{aligned}
$$

Combining estimates (3.14) and (3.15), the proof of (3.9) is complete.

The bound (3.10) on the ground state energy follows from (3.9) simply by taking the infimum over all $\Omega \subset Q_{L}$ with $|\Omega|=\vartheta L^{3}$. The proof of Lemma 3.3 is therefore complete.

Using Lemmas 3.1 and 3.3, we are now ready to give the proof of the upper bound from Proposition 2.1 without assuming any symmetry on $\Omega^{*}$. Since most parts are identical to the proof in section 2 , we only give the necessary modifications in the construction of the competitor set at the beginning of the proof.

Proof of Proposition 2.1 without symmetry of $\Omega^{*}$. As in the proof given in section 2 , let us set $l_{0}:=A^{* 1 / 3} \vartheta^{-1 / 3}$ to be the characteristic length of the small boxes. Let $\Omega^{*}$ be some set satisfying $\left|\Omega^{*}\right|=A^{*}$ and $\mathcal{E}\left[\Omega^{*}\right]=E\left(A^{*}\right)$. We may assume (up to changing $\Omega^{*}$ on a null-set) that $\operatorname{diam}\left(\Omega^{*}\right)<\infty$; see [15, Lemma 4.1] and [19, Lemma 4]. By Lemma 3.1, there are $U \in \mathbb{R}^{3 \times 3}$ orthogonal, $y \in \mathbb{R}^{3}$ and $\boldsymbol{\lambda} \in \mathbb{R}^{3}$ with $\left|\lambda_{i}-1\right| \leq C l_{0}^{-2}$, and $\lambda_{1} \lambda_{2} \lambda_{3}=1$ such that setting $\boldsymbol{l}=\boldsymbol{\lambda} l_{0}$, the set $\Omega_{0}^{*}:=U\left(\Omega^{*}+y\right)$ is contained in $Q_{l}$ and satisfies

$0=\int_{\mathbb{R}^{3}}\left(1_{\Omega_{0}^{*}}(x)-\vartheta 1_{Q_{l}}\right) \mathrm{d} x=\int_{\mathbb{R}^{3}} x_{i}\left(1_{\Omega_{0}^{*}}(x)-\vartheta 1_{Q_{l}}\right) \mathrm{d} x=\int_{\mathbb{R}^{3}}\left(3 x_{i} x_{j}-\delta_{i j}|x|^{2}\right)\left(1_{\Omega_{0}^{*}}(x)-\vartheta 1_{Q_{l}}\right) \mathrm{d} x$.

By Lemma 3.3, we have

$$
E_{\vartheta, L} \leq\left(1+C l_{0}^{-2}\right) E_{\vartheta, L, \boldsymbol{\lambda}}=\left(1+C \vartheta^{2 / 3}\right) E_{\vartheta, L, \boldsymbol{\lambda}}
$$

To prove Proposition 2.1, it therefore suffices to prove the upper bound

$$
\frac{E_{\vartheta, L, \boldsymbol{\lambda}}}{\vartheta L^{3}} \leq \frac{E\left(A^{*}\right)}{A^{*}}+C \vartheta^{1 / 3}+\frac{C}{\vartheta^{1 / 3} L}
$$


because the additional error term coming from the estimate (3.17) is subleading.

To prove (3.18), we construct a competitor set by placing copies of the set $\Omega_{0}^{*}$ in boxes $Q_{l}(r), r \in \mathbb{Z}^{3}$. Let $\mathcal{C}_{l}=\left\{r \in \mathbb{Z}^{3}: Q_{l}(r) \subset \lambda Q_{L}\right\}$ be the set of lattice points $r$ such that the cubes $Q_{l}(r)$ are fully contained in $\lambda Q_{L}$. Then, setting

$$
\lambda_{\vartheta, L, \boldsymbol{\lambda}}^{3}=\frac{\vartheta L^{3}}{A^{*}\left|\mathcal{C}_{l}\right|}
$$

we obtain $\lambda Q_{L}$ as a union of the boxes $Q_{\lambda_{\vartheta, L, \lambda} l_{0}}(r)$. That is, we can cover the large box exactly by an integer number of small boxes. We therefore define

$$
\Omega_{\vartheta, L, \boldsymbol{\lambda}}=\bigcup_{r \in \mathcal{C}_{l}}\left(\lambda_{\vartheta, L, \boldsymbol{\lambda}} \Omega_{0}^{*}+\boldsymbol{l} r\right), \quad \text { where } \quad \boldsymbol{l} r:=\left(l_{1} r_{1}, l_{2} r_{2}, l_{3} r_{3}\right) .
$$

Note that this definition fulfills the mass constraint

$$
\left|\Omega_{\vartheta, L}\right|=\left|\mathcal{C}_{l}\right| A^{*} \lambda_{\vartheta, L}^{3}=\vartheta L^{3}
$$

The proof of Proposition 2.1 can now be finalized by following exactly the same steps as in section 2, using the vanishing of the multipole moments from (3.16) in the bound on the far-field interaction. We omit the remaining details.

4. Lower bound on the ground state energy. In this section, we give the proof of the lower bound from Theorem 1.1. Again, we restate here for convenience the result we want to prove.

Proposition 4.1 (Lower bound). There is a constant $C>0$ such that for all $\vartheta \in(0,1], L>0$

$$
\frac{E_{\vartheta, L}}{\vartheta L^{3}} \geq \frac{E\left(A^{*}\right)}{A^{*}}-C \vartheta^{1 / 5} .
$$

The proof of Proposition 4.1 is based on reducing the problem to a smaller length scale $1 \ll R \ll L$.

We define the Yukawa potential

$$
Y_{\omega}(x)=\frac{\mathrm{e}^{-\omega|x|}}{|x|} \quad \text { for } \quad x \in \mathbb{R}^{3} \quad \text { and } \quad \omega>0 .
$$

Using $Y_{\omega}$, we can bound the interaction part of $\mathcal{E}_{\vartheta, L}[\Omega]$ from below as follows.

LEMma 4.2 (Lower bound on the interaction term). There is $C>0$ such that for all $L>0$, all $\vartheta \in[0,1]$, all $\omega>0$, and all $\Omega \subset Q_{L}$, we have

$$
\begin{aligned}
& \int_{\mathbb{R}^{3}} \int_{\mathbb{R}^{3}}\left(1_{\Omega}(x)-\vartheta 1_{Q_{L}}(x)\right) \frac{1}{|x-y|}\left(1_{\Omega}(y)-\vartheta 1_{Q_{L}}(y)\right) \mathrm{d} x \mathrm{~d} y \\
\geq & \int_{\mathbb{R}^{3}} 1_{\Omega}(x) Y_{\omega}(x-y) 1_{\Omega}(y) \mathrm{d} x \mathrm{~d} y-C|\Omega| \vartheta \omega^{-2} .
\end{aligned}
$$

Proof. First, we can estimate

$$
\begin{gathered}
\int_{\mathbb{R}^{3}} \int_{\mathbb{R}^{3}}\left(1_{\Omega}(x)-\vartheta 1_{Q_{L}}(x)\right) \frac{1}{|x-y|}\left(1_{\Omega}(y)-\vartheta 1_{Q_{L}}(y)\right) \mathrm{d} x \mathrm{~d} y \\
\geq \int_{\mathbb{R}^{3}} \int_{\mathbb{R}^{3}}\left(1_{\Omega}(x)-\vartheta 1_{Q_{L}}(x)\right) Y_{\omega}(x-y)\left(1_{\Omega}(y)-\vartheta 1_{Q_{L}}(y)\right) \mathrm{d} x \mathrm{~d} y,
\end{gathered}
$$


because $\mathcal{F}\left[\frac{1}{|x|}\right]=\sqrt{\frac{\pi}{2}} \frac{1}{|k|^{2}} \geq \sqrt{\frac{\pi}{2}} \frac{1}{|k|^{2}+\omega^{2}}=\mathcal{F}\left[Y_{\omega}\right](k)$, where $\mathcal{F}$ denotes the Fourier transform. Next,

$$
\begin{aligned}
& \int_{\mathbb{R}^{3}} \int_{\mathbb{R}^{3}}\left(1_{\Omega}(x)-\vartheta 1_{Q_{L}}(x)\right) Y_{\omega}(x-y)\left(1_{\Omega}(y)-\vartheta 1_{Q_{L}}(y)\right) \mathrm{d} x \mathrm{~d} y \\
& \geq \int_{\mathbb{R}^{3}} \int_{\mathbb{R}^{3}} 1_{\Omega}(x) Y_{\omega}(x-y) 1_{\Omega}(y) \mathrm{d} x \mathrm{~d} y-2 \vartheta \int_{\mathbb{R}^{3}} \int_{\mathbb{R}^{3}} 1_{\Omega}(x) Y_{\omega}(x-y) 1_{Q_{L}}(y) \mathrm{d} x \mathrm{~d} y
\end{aligned}
$$

$$
\geq \int_{\mathbb{R}^{3}} \int_{\mathbb{R}^{3}} 1_{\Omega}(x) Y_{\omega}(x-y) 1_{\Omega}(y) \mathrm{d} x \mathrm{~d} y-C|\Omega| \vartheta \omega^{-2},
$$

where we bounded $\int_{Q_{L}} Y_{\omega}(x-y) \mathrm{d} y \leq \int_{\mathbb{R}^{3}} \frac{e^{-\omega|y|}}{|y|} \mathrm{d} y \leq C \omega^{-2}$.

We also need to control the behavior of the perimeter term under localization of $\Omega \subset Q_{L}$ to smaller boxes. The following lemma is useful for this purpose.

LEMMA 4.3 (Localization of the perimeter term). Let $\Omega \subset \mathbb{R}^{3}$ have finite perimeter. Then for every $R>0$,

$$
\operatorname{Per}(\Omega) \geq \sum_{m \in \mathbb{Z}^{3}} \int_{Q_{1}} \operatorname{Per}\left(\Omega \cap Q_{R}(m+\xi)\right) \mathrm{d} \xi-\frac{6|\Omega|}{R}
$$

Proof. In every box, the boundary of $\Omega \cap Q_{R}(m+\xi)$ consists of two parts: the portion of $\partial \Omega$ lying inside $Q_{R}(m+\xi)$, and the portion of $\Omega$ intersecting $\partial Q_{R}(m+\xi)$, which is added by partitioning $\Omega$ into boxes. We therefore have that

$$
\begin{aligned}
& \sum_{m \in \mathbb{Z}^{3}} \int_{Q_{1}} \operatorname{Per}\left(\Omega \cap Q_{R}(m+\xi)\right) \mathrm{d} \xi \\
& \leq \int_{Q_{1}} \sum_{m \in \mathbb{Z}^{3}} \mathcal{H}^{2}\left(\partial \Omega \cap Q_{R}(m+\xi)\right) \mathrm{d} \xi+\int_{Q_{1}} \sum_{m \in \mathbb{Z}^{3}} \mathcal{H}^{2}\left(\Omega \cap \partial Q_{R}(m+\xi)\right) \mathrm{d} \xi \\
& \leq \operatorname{Per}(\Omega)+\int_{Q_{1}} \sum_{m \in \mathbb{Z}^{3}} \mathcal{H}^{2}\left(\Omega \cap \partial Q_{R}(m+\xi)\right) \mathrm{d} \xi .
\end{aligned}
$$

Here, $\mathcal{H}^{2}$ denotes the two-dimensional Hausdorff measure. It remains to evaluate the second term in (4.3). Since all sets appearing there are subsets of faces of cubes, we can decompose

$$
\bigcup_{m \in \mathbb{Z}^{3}} \Omega \cap \partial Q_{R}(m+\xi)=\bigcup_{i=1}^{3} \bigcup_{l \in \mathbb{Z}} \Omega \cap\left\{x \in \mathbb{R}^{3}: x_{i}=R\left(l+\frac{1}{2}+\xi_{i}\right)\right\}
$$

i.e., we distinguish the "slices" of $\Omega \cap \partial Q_{R}(m+\xi)$ according to the coordinate hyperplane they are parallel to. Note that $\mathcal{H}^{2}$-almost every point in one hyperplane is contained in the boundary of exactly two cubes adjacent to the plane. Thus the union $\bigcup_{i=1}^{3}$ is disjoint up to an $\mathcal{H}^{2}$-null-set, and we obtain

$\int_{Q_{1}} \sum_{m \in \mathbb{Z}^{3}} \mathcal{H}^{2}\left(\Omega \cap \partial Q_{R}(m+\xi)\right) \mathrm{d} \xi=2 \sum_{i=1}^{3} \int_{[-1 / 2,1 / 2]^{3}} \mathrm{~d} \xi_{1} \mathrm{~d} \xi_{2} \mathrm{~d} \xi_{3} \sum_{l \in \mathbb{Z}} \mathcal{H}^{2}\left(\Omega \cap\left\{x_{i}=R\left(l+1 / 2+\xi_{i}\right)\right\}\right)$.

The integrand on the right-hand side only depends on one of the $\xi_{i}$. We can therefore 
do the $\mathrm{d} \xi_{j}$-integrations with $j \neq i$ to find that

$$
\begin{aligned}
& \int_{Q_{1}} \sum_{m \in \mathbb{Z}^{3}} \mathcal{H}^{2}\left(\Omega \cap \partial Q_{R}(m+\xi)\right) \mathrm{d} \xi=2 \sum_{i=1}^{3} \int_{-1 / 2}^{1 / 2} \sum_{l \in \mathbb{Z}} \mathcal{H}^{2}\left(\Omega \cap\left\{x_{i}=R\left(l+1 / 2+\xi_{i}\right)\right\}\right) \mathrm{d} \xi_{i} \\
& =2 \sum_{i=1}^{3} \int_{\mathbb{R}} \mathcal{H}^{2}\left(\Omega \cap\left\{x_{i}=R \xi_{i}\right\}\right) \mathrm{d} \xi_{i}=\frac{2}{R} \sum_{i=1}^{3} \int_{\mathbb{R}} \mathcal{H}^{2}\left(\Omega \cap\left\{x_{i}=\xi_{i}\right\}\right) \mathrm{d} \xi_{i}=\frac{6|\Omega|}{R}
\end{aligned}
$$

by Fubini's theorem. Plugging this into (4.3) completes the proof of Lemma 4.3.

In the next lemma we combine the above estimates to obtain the crucial lower bound on the energy in terms of the auxiliary parameters $R$ and $\omega$.

Lemma 4.4. For every $\Omega \subset Q_{L}$ with $|\Omega|>0$ and every $R>0$, we have that

$$
\frac{\mathcal{E}_{\vartheta, L}[\Omega]}{|\Omega|} \geq e^{-\sqrt{3} \omega R} \frac{E\left(A^{*}\right)}{A^{*}}-C \vartheta \omega^{-2}-\frac{6}{R} .
$$

Proof. Let $\Omega \subset Q_{L}$ and $R>0$. We split $\Omega$ into the (finite) disjoint union

$$
\Omega=\bigcup_{m \in \mathbb{Z}^{3}}\left(\Omega \cap Q_{R}\left(m+\xi_{0}\right)\right)=: \bigcup_{m \in \mathbb{Z}^{3}} \Omega^{(m)}
$$

for some $\xi_{0} \in Q_{1}$ to be chosen below. Note that our choice of $\Omega^{(m)}$ in (4.4) ensures that $\operatorname{diam}\left(\Omega^{(m)}\right) \leq \sqrt{3} R$.

Hence, starting from Lemma 4.2 and dropping the interactions between different boxes, we can estimate the energy from below as follows:

$$
\begin{aligned}
\mathcal{E}_{\vartheta, L}[\Omega] & \geq \sum_{m \in \mathbb{Z}^{3}}\left(\operatorname{Per}\left(\Omega^{(m)}\right)+e^{-\omega \sqrt{3} R} \frac{1}{2} \iint_{\Omega^{(m)} \times \Omega^{(m)}} \frac{\mathrm{d} x \mathrm{~d} y}{|x-y|}\right)+\mathcal{P}_{R}-C|\Omega| \vartheta \omega^{-2} \\
& \geq e^{-\sqrt{3} \omega R} \sum_{m \in \mathbb{Z}^{3}} \mathcal{E}\left[\Omega^{(m)}\right]+\mathcal{P}_{R}-C|\Omega| \vartheta \omega^{-2}
\end{aligned}
$$

with the perimeter error term $\mathcal{P}_{R}:=\operatorname{Per}(\Omega)-\sum_{m \in \mathbb{Z}} \operatorname{Per}\left(\Omega^{(m)}\right)$.

For every $m \in \mathbb{Z}^{3}$ with $\left|\Omega^{(m)}\right|>0$, we have $\frac{\mathcal{E}\left[\Omega^{(m)}\right]}{\left|\Omega^{(m)}\right|} \geq \frac{E\left(A^{*}\right)}{A^{*}}$, and hence

$$
\sum_{m \in \mathbb{Z}^{3}} \mathcal{E}\left[\Omega^{(m)}\right]=\sum_{m \in \mathbb{Z}^{3},\left|\Omega^{(m)}\right|>0} \frac{\mathcal{E}\left[\Omega^{(m)}\right]}{\left|\Omega^{(m)}\right|}\left|\Omega^{(m)}\right| \geq \frac{E\left(A^{*}\right)}{A^{*}} \sum_{m \in \mathbb{Z}^{3}}\left|\Omega^{(m)}\right|=|\Omega| \frac{E\left(A^{*}\right)}{A^{*}} .
$$

Together with (4.6), the lower bound (4.5) implies

$$
\mathcal{E}_{\vartheta, L}[\Omega] \geq e^{-\sqrt{3} \omega R} \frac{E\left(A^{*}\right)}{A^{*}}|\Omega|+\mathcal{P}_{R}-C|\Omega| \vartheta \omega^{-2} .
$$

To bound the perimeter error $\mathcal{P}_{R}$ appropriately, recall from Lemma 4.3 that we have the averaged estimate

$$
\int_{Q_{1}}\left(\sum_{m \in \mathbb{Z}^{3}} \operatorname{Per}\left(\Omega \cap Q_{R}(m+\xi)\right)\right) \mathrm{d} \xi \leq \operatorname{Per}(\Omega)+\frac{6|\Omega|}{R},
$$

and therefore there exists $\xi_{0} \in Q_{1}$ depending on $\Omega$ such that

$$
\sum_{m \in \mathbb{Z}^{3}} \operatorname{Per}\left(\Omega \cap Q_{R}\left(m+\xi_{0}\right)\right) \leq \operatorname{Per}[\Omega]+\frac{6|\Omega|}{R} .
$$


With this choice of $\xi_{0}$, we arrive at the bound

$$
\mathcal{P}_{R}=\operatorname{Per}(\Omega)-\sum_{m \in \mathbb{Z}} \operatorname{Per}\left(\Omega^{(m)}\right) \geq-\frac{6|\Omega|}{R} .
$$

Combining (4.7) and (4.10) and dividing by $|\Omega|$, the statement of Lemma 4.4 follows. $\square$

It only remains to minimize the errors of the lower bound to the ground state energy $E_{\vartheta, L}$.

Proof of Proposition 4.1. Recalling that $|\Omega|=\vartheta L^{3}$, by Lemma 4.4 we have

$$
\frac{E_{\vartheta, L}}{\vartheta L^{3}} \geq e^{-\sqrt{3} \omega R} \frac{E\left(A^{*}\right)}{A^{*}}-C \vartheta \omega^{-2}-\frac{6}{R} .
$$

Since $e^{-x} \geq 1-x$, from (4.11) we obtain

$$
\begin{aligned}
\frac{E_{\vartheta, L}}{\vartheta L^{3}} & \geq(1-\sqrt{3} \omega R) \frac{E\left(A^{*}\right)}{A^{*}}-C \vartheta \omega^{-2}-\frac{C}{R} \\
& \geq \frac{E\left(A^{*}\right)}{A^{*}}-C \omega R-\frac{C}{R}-C \vartheta \omega^{-2} .
\end{aligned}
$$

Optimizing first in $R$, we take $R=\omega^{-1 / 2}$. With that choice, we have the inequality

$$
\frac{E_{\vartheta, L}}{\vartheta L^{3}} \geq \frac{E\left(A^{*}\right)}{A^{*}}-C \omega^{1 / 2}-C \vartheta \omega^{-2} .
$$

Optimizing in $\omega$ gives $\omega=\vartheta^{2 / 5}$, and hence we get

$$
\frac{E_{\vartheta, L}}{\vartheta L^{3}} \geq \frac{E\left(A^{*}\right)}{A^{*}}-C \vartheta^{1 / 5}
$$

The proof of Proposition 4.1 is now complete.

5. Independence of the thermodynamic limit from boundary conditions. In this section we prove Theorem 1.5. Although we are confident that in our setting the existence of the thermodynamic limit could be proved with the classical methods from $[17,18]$, it seems hard to study different boundary conditions or obtain good asymptotics in this framework. We therefore rely here strongly on a more recent result by Alberti, Choksi, and Otto [1], who prove convergence of the energy per volume with an essentially optimal convergence rate of $L^{-1}$ in the case of Dirichlet and Neumann boundary conditions. We show that their conclusion also holds for periodic boundary conditions, as well as for the "whole space" boundary conditions which we use in this paper. In fact, we will see that the Dirichlet and Neumann boundary conditions give the lowest, respectively, highest, interaction energy to any set $\Omega$ among a large class of boundary conditions; see Lemmas 5.1 and 5.2 and Remark 5.3 below.

Throughout the following, we fix some $\vartheta \in(0,1)$ which will not be reflected in the notation. We also recall the definitions (1.9)-(1.11) for $\#=P, D, N, \infty$.

The proofs in this section rely on rewriting the interaction energy in (1.9) as a gradient integral. This is inspired by the approach in [1]. Given an admissible set $\Omega \subset Q_{L}$ with $|\Omega|=\vartheta L^{3}$, we thus define

$$
v_{\#, L}^{(\Omega)}(x):=\int_{Q_{L}} G_{\#, L}(x, y)\left(1_{\Omega}(y)-\vartheta\right) \mathrm{d} y, \quad x \in Q_{L}, \quad \text { for } \# \in\{D, N, P\}
$$


and

$$
v_{\infty, L}^{(\Omega)}(x):=\frac{1}{4 \pi} \int_{Q_{L}} \frac{1_{\Omega}(y)-\vartheta}{|x-y|} \mathrm{d} y, \quad x \in \mathbb{R}^{3}, \quad \text { for } \#=\infty,
$$

the potential associated with $\Omega$. The function $v_{\#, L}^{(\Omega)}$ satisfies

$$
-\Delta v_{\#, L}^{(\Omega)}=1_{\Omega}-\vartheta \quad \text { on } Q_{L}
$$

together with the boundary conditions given by the choice of \#. Notice that we even have

$$
-\Delta v_{\infty}^{(\Omega)}=1_{\Omega}-\vartheta 1_{Q_{L}} \quad \text { on all of } \mathbb{R}^{3} .
$$

Using integration by parts together with the respective boundary conditions, we can now write the energy $\mathcal{E}_{\#, L}[\Omega]$ of any set $\Omega \subset Q_{L}$ with $|\Omega|=\vartheta L^{3}$ as

$$
\mathcal{E}_{\#, L}[\Omega]=\operatorname{Per}(\Omega)+\frac{1}{2} \int_{Q_{L}}\left|\nabla v_{\#, L}^{(\Omega)}(x)\right|^{2} \mathrm{~d} x \quad \text { for } \#=D, N, P,
$$

respectively,

$$
\mathcal{E}_{\infty, L}[\Omega]=\operatorname{Per}(\Omega)+\frac{1}{2} \int_{\mathbb{R}^{3}}\left|\nabla v_{\infty, L}^{(\Omega)}(x)\right|^{2} \mathrm{~d} x \quad \text { for } \#=\infty .
$$

The inequalities contained in the following two lemmas are needed for the proof of Theorem 1.5.

LEMMA 5.1. Let $L>0$ and $\Omega \subset Q_{L}$ with $|\Omega|=\vartheta L^{3}$. Then $\mathcal{E}_{D, L}[\Omega] \leq \mathcal{E}_{\#, L}[\Omega]$ for all $\# \in\{N, P, \infty\}$.

Proof of Lemma 5.1. Let $\Omega \subset Q_{L}$ with $|\Omega|=\vartheta L^{3}$, let $\# \in\{N, P, \infty\}$, and abbreviate $v_{D}=v_{D, L}^{(\Omega)}$ and $v_{\#}=v_{\#, L}^{(\Omega)}$. Then we have

$$
\begin{aligned}
\int_{Q_{L}}\left|\nabla v_{\#}\right|^{2} & =\int_{Q_{L}}\left|\nabla v_{D}\right|^{2}+\int_{Q_{L}}\left|\nabla\left(v_{D}-v_{\#}\right)\right|^{2}-2 \int_{Q_{L}}\left(\nabla v_{D}-\nabla v_{\#}\right) \cdot \nabla v_{D} \\
& =\int_{Q_{L}}\left|\nabla v_{D}\right|^{2}+\int_{Q_{L}}\left|\nabla\left(v_{D}-v_{\#}\right)\right|^{2}-2 \int_{\partial Q_{L}} \frac{\partial\left(v_{D}-v_{\#}\right)}{\partial \nu} v_{D} \geq \int_{Q_{L}}\left|\nabla v_{D}\right|^{2},
\end{aligned}
$$

where we used $\Delta v_{D}=\Delta v_{\#}$ on $Q_{L}$ and the fact that $v_{D}$ vanishes on $\partial Q_{L}$. We conclude that

$$
\mathcal{E}_{D, L}[\Omega]=\operatorname{Per}(\Omega)+\int_{Q_{L}}\left|\nabla v_{D}\right|^{2} \leq \operatorname{Per}(\Omega)+\int_{Q_{L}}\left|\nabla v_{\#}\right|^{2} \leq \mathcal{E}_{\#, L}[\Omega]
$$

since the perimeter term does not depend on the boundary condition.

LEMMA 5.2. Let $L>0$ and $\Omega \subset Q_{L}$ with $|\Omega|=\vartheta L^{3}$. Then $\mathcal{E}_{\#, L}[\Omega] \leq \mathcal{E}_{N, L}[\Omega]$ for all $\# \in\{D, P, \infty\}$.

Proof. Let $\Omega \subset Q_{L}$ with $|\Omega|=\vartheta L^{3}$, let $\# \in\{D, P, \infty\}$, and abbreviate $v_{\#}=v_{\#, L}^{(\Omega)}$ and $v_{N}=v_{N, L}^{(\Omega)}$. Performing the same calculation as in the proof of Lemma 5.1, we obtain

$$
\begin{aligned}
\int_{Q_{L}}\left|\nabla v_{N}\right|^{2} & =\int_{Q_{L}}\left|\nabla v_{\#}\right|^{2}+\int_{Q_{L}}\left|\nabla\left(v_{\#}-v_{N}\right)\right|^{2}-2 \int_{Q_{L}}\left(\nabla v_{\#}-\nabla v_{N}\right) \cdot \nabla v_{\#} \\
& =\int_{Q_{L}}\left|\nabla v_{\#}\right|^{2}+\int_{Q_{L}}\left|\nabla\left(v_{\#}-v_{N}\right)\right|^{2}-2 \int_{\partial Q_{L}} \frac{\partial v_{\#}}{\partial \nu} v_{\#},
\end{aligned}
$$


where we used $\Delta v_{N}=\Delta v_{\#}$ on $Q_{L}$ and the fact that $\frac{\partial v_{N}}{\partial \nu}=0$ on $\partial Q_{L}$. The Dirichlet and the periodic boundary conditions imply that

$$
-\int_{\partial Q_{L}} \frac{\partial v_{D}}{\partial \nu} v_{D}=-\int_{\partial Q_{L}} \frac{\partial v_{P}}{\partial \nu} v_{P}=0
$$

Therefore, we deduce from (5.4) the bound

$$
\int_{Q_{L}}\left|\nabla v_{N}\right|^{2} \geq \int_{Q_{L}}\left|\nabla v_{\#}\right|^{2}
$$

for $\# \in\{D, P\}$. In case $\#=\infty$, integration by parts yields

$$
-2 \int_{\partial Q_{L}} \frac{\partial v_{\infty}}{\partial \nu} v_{\infty}=2 \int_{\mathbb{R}^{3} \backslash Q_{L}}\left|\nabla v_{\infty}\right|^{2} \geq 0,
$$

since $\Delta v_{\infty}=0$ on $\mathbb{R}^{3} \backslash Q_{L}$. Plugging this into (5.4), we get

$$
\int_{Q_{L}}\left|\nabla v_{N}\right|^{2} \geq \int_{\mathbb{R}^{3}}\left|\nabla v_{\infty}\right|^{2}
$$

The lemma follows since the perimeter term does not depend on the boundary condition.

Remark 5.3. We point out that the validity of Lemmas 5.1 and 5.2 requires neither minimization nor passage to the thermodynamic limit. Moreover, the proofs of those lemmas show that we can treat in the same way any self-adjoint boundary condition on $\partial Q_{L}$ with the property that $-\int_{\partial Q_{L}} \frac{\partial v}{\partial \nu} v \geq 0$ for every $v$ satisfying this boundary condition. A large such class is given, for example, by the Robin boundary condition

$$
\frac{\partial v}{\partial \nu}(x)=-\beta\left(\frac{x}{L}\right) v(x) \quad \text { on } \partial Q_{L},
$$

where $\beta \in L^{\infty}\left(\partial Q_{1}\right)$ is an arbitrary nonnegative bounded function on $\partial Q_{1}$.

The proof of Theorem 1.5 now follows by a simple combination of Lemmas 5.1 and 5.2 with the results from [1].

Proof of Theorem 1.5. It has been proved in [1] that there exist $\sigma^{*}>0$ and $C>0$ such that

$$
\sigma^{*}-\frac{C}{L} \leq \frac{E_{D, L}(\vartheta)}{L^{3}} \leq \frac{E_{N, L}(\vartheta)}{L^{3}} \leq \sigma^{*}+\frac{C}{L}
$$

for all $L \geq C$. Moreover, we have

$$
\mathcal{E}_{D, L}[\Omega] \leq \mathcal{E}_{\#, L}[\Omega] \quad \text { for all } \quad \# \in\{N, P, \infty\}
$$

by Lemma 5.1 and

$$
\mathcal{E}_{\#, L}[\Omega] \leq \mathcal{E}_{N, L}[\Omega] \quad \text { for all } \quad \# \in\{D, P, \infty\}
$$

by Lemma 5.2. Theorem 1.5 follows as an immediate consequence of the above inequalities. 


\section{REFERENCES}

[1] G. Alberti, R. Choksi, And F. Отto, Uniform energy distribution for an isoperimetric problem with long-range interactions, J. Amer. Math. Soc., 22 (2009), pp. 569-605, https://doi.org/10.1090/S0894-0347-08-00622-X.

[2] M. BONACINI AND R. CRISTOFERI, Local and global minimality results for a nonlocal isoperimetric problem on $\mathbb{R}^{N}$, SIAM J. Math. Anal., 46 (2014), pp. 2310-2349, https://doi.org/ $10.1137 / 130929898$.

[3] R. Choksi, C. B. Muratov, and I. Topaloglu, An old problem resurfaces nonlocally: Gamow's liquid drops inspire today's research and applications, Notices Amer. Math. Soc., 64 (2017), pp. 1275-1283.

[4] R. Choksi And M. A. Peletier, Small volume fraction limit of the diblock copolymer problem: I. Sharp-interface functional, SIAM J. Math. Anal., 42 (2010), pp. 1334-1370, https://doi. org/10.1137/090764888.

[5] R. ChOKSi AND M. A. Peletien, Small volume-fraction limit of the diblock copolymer problem: II. Diffuse-interface functional, SIAM J. Math. Anal., 43 (2011), pp. 739-763, https://doi. org $/ 10.1137 / 10079330 X$.

[6] S. DANERI AND E. RUNA, Exact periodic stripes for minimizers of a local/nonlocal interaction functional in general dimension, Arch. Ration. Mech. Anal., 231 (2019), pp. 519-589, https://doi.org/10.1007/s00205-018-1285-6.

[7] R. L. Frank And E. H. Lieb, A compactness lemma and its application to the existence of minimizers for the liquid drop model, SIAM J. Math. Anal., 47 (2015), pp. 4436-4450, https://doi.org/10.1137/15M1010658.

[8] R. L. Frank and E. H. Lieb, Periodic energy minimizers for a one-dimensional liquid drop model, Lett. Math. Phys., 109 (2019), pp. 2069-2081.

[9] G. Gamow, Mass defect curve and nuclear constitution, Proc. R. Soc. Lond. A, 126 (1930), pp. 632-644.

[10] A. Giuliani And R. Seiringer, Periodic striped ground states in Ising models with competing interactions, Comm. Math. Phys., 347 (2016), pp. 983-1007, https://doi.org/10.1007/ s00220-016-2665-0.

[11] M. Goldman And E. Runa, On the optimality of stripes in a variational model with non-local interactions, Calc. Var. Partial Differential Equations, 58 (2019), 103.

[12] M.-A. Hashimoto, H. Seki, And M. Yamada, Shape of nuclei in the crust of neutron star, Progr. Theoret. Phys., 71 (1984), pp. 320-326.

[13] V. Julin, Isoperimetric problem with a Coulomb repulsive term, Indiana Univ. Math. J., 63 (2014), pp. 77-89, https://doi.org/10.1512/iumj.2014.63.5185.

[14] V. Julin, Remark on a nonlocal isoperimetric problem, Nonlinear Anal., 154 (2017), pp. 174188, https://doi.org/10.1016/j.na.2016.10.011.

[15] H. KNüPfER AND C. B. Muratov, On an isoperimetric problem with a competing nonlocal term II: The general case, Comm. Pure Appl. Math., 67 (2014), pp. 1974-1994, https: //doi.org/10.1002/cpa.21479.

[16] H. Knüpfer, C. B. Muratov, and M. Novaga, Low density phases in a uniformly charged liquid, Comm. Math. Phys., 345 (2016), pp. 141-183, https://doi.org/10.1007/ s00220-016-2654-3.

[17] E. H. Lieb AND J. L. Lebowitz, The constitution of matter: Existence of thermodynamics for systems composed of electrons and nuclei, Advances in Math., 9 (1972), pp. 316-398, https://doi.org/10.1016/0001-8708(72)90023-0.

[18] E. H. Lieb and H. Narnhofer, The thermodynamic limit for jellium, J. Statist. Phys., 12 (1975), pp. 291-310, https://doi.org/10.1007/BF01012066.

[19] J. LU AND F. Отто, Nonexistence of a minimizer for Thomas-Fermi-Dirac-von Weizsäcker model, Comm. Pure Appl. Math., 67 (2014), pp. 1605-1617, https://doi.org/10.1002/cpa. 21477 .

[20] K. Oyamatsu, M.-A. Hashimoto, and M. Yamada, Further study of the nuclear shape in high-density matter, Progr. Theoret. Phys., 72 (1984), pp. 373-375, https://doi.org/10. 1143/PTP.72.373.

[21] D. Ravenhall, C. Pethick, And J. Wilson, Structure of matter below nuclear saturation density, Phys. Rev. Lett., 50 (1983), 2066. 